\title{
Multi-objective flower pollination algorithm applied to 5G vehicular networks
}

\section{communication}

\author{
Algoritmo multiobjetivo de polinização de flores aplicado à comunicação de redes veiculares $5 \mathrm{G}$ \\ Algoritmo multiobjetivo de polinización de flores aplicado a comunicación de redes vehiculares 5G
}

\begin{abstract}
The Cellular Vehicle-to-Everything (C-V2X) technology, as a widest version of Vehicular Ad-hoc Network (VANET), aims to interconnect vehicles and any other latest technological infrastructures. In this context, the fifth generation of mobile networks (5G) based on millimeter waves (mmWave) is an excellent alternative for the implementation of vehicular networks, mainly because it is capable of providing high data rates (Gbps) and ultra-low latency, requirements of C-V2X. On the other hand, mmWave signals are highly susceptible to blocking, causing low quality of service (QoS) in VANETs, compromising network functionality and the safety of drivers and pedestrians. Thus, in this work evolutionary computing techniques are applied in the simulation of a $5 \mathrm{G}$ vehicular network based on millimeter waves, exploring Media Access Control (MAC) sublayer parameters to optimize packet loss, latency and throughput, in order to optimize inter-vehicular communication. The Multi-objective Flower Pollination Algorithm (MOFPA) was used for this purpose. The results obtained show that the adopted approach can reach results close to the optimal pareto of non-dominated solutions, with a 75\% reduction in exploration time in relation to the exhaustive search process. Finally, the performance of the metaheuristics adopted is compared with the non-dominated genetic classification algorithm (NSGA-II) and the multi-objective differential evolutionary algorithm (MODE).
\end{abstract}

Keywords: Vehicular networks; 5G; mmWave; Evolutionary computation; Flower pollination algorithm.

\section{Resumo}

A tecnologia Cellular Vehicle-to-Everything (C-V2X), versão mais ampla das VANETs (Veicular Ad Hoc Networks), pretende interligar veículos e quaisquer outras infraestruturas tecnológicas de última geração. Nesse contexto a quinta geração de redes móveis (5G) baseada em ondas milimétricas (mmWave) é uma excelente alternativa para implantação de redes veiculares, principalmente por ser capaz de fornecer altas taxas de dados (Gbps) e latência ultrabaixa, requisitos do C-V2X. Por outro, lado sinais mmWave são altamente suscetíveis ao bloqueio, o que provoca baixa qualidade de serviço (QoS) nas VANETs, comprometendo as funcionalidades da rede e a segurança de motoristas e pedestres. Dessa forma este trabalho aplicou técnicas de computação evolutiva na simulação de uma rede veicular 5G, baseada em ondas milimétricas, explorando parâmetros de controle da camada de acesso ao meio (MAC) para otimizar a perda de pacotes, latência e throughput, com o objetivo de otimizar a comunicação interveicular. Para esse propósito foi utilizado O Algoritmo Multiobjetivo de Polinização de Flores (MOFPA). Os resultados obtidos mostram que a abordagem adotada pode atingir resultados próximos ao pareto ótimo de soluções não dominadas, com redução de $75 \%$ no tempo de exploração em comparação com o processo de busca exaustiva. Por fim, o desempenho 
da metaheurística adotava foi comparado com o non-dominated genetic classification algorithm (NSGA-II) e o multiobjective differential evolutionary algorithm (MODE).

Palavras-chave: Redes veiculares; 5G; mmWave; Computação evolutiva; Algoritmo de polinização de flores.

\section{Resumen}

La tecnología celular vehículo-a-todo (C-V2X), una versión más amplia de la red ad-hoc vehicular (VANET), tiene como objetivo interconectar vehículos y cualquier otra infraestructura tecnológica vanguardista. En este contexto, la quinta generación de redes móviles $(5 \mathrm{G})$ basadas en ondas milimétricas (mmWave) es una excelente alternativa para la implementación de redes vehiculares, principalmente porque es capaz de brindar altas tasas de datos (Gbps) y latencia ultrabaja, requisitos del estándar C-V2X. Por otro lado, las señales mmWave son altamente susceptibles a bloqueos, lo que provoca una baja calidad de servicio (QoS) en los VANETs, comprometiendo la funcionalidad de la red y la seguridad de conductores y peatones. Así, en este trabajo se aplican técnicas de computación evolutiva en la simulación de una red vehicular $5 \mathrm{G}$ basada en ondas milimétricas, explorando los parámetros de la subcapa de control de acceso al medio (MAC) para optimizar la pérdida de paquetes, la latencia y la tasa de transferencia efectiva, con el fin de optimizar la comunicación inter-vehicular. Para este propósito se utilizó el Algoritmo de Polinización de Flores Multiobjetivo (MOFPA). Los resultados obtenidos muestran que el enfoque adoptado puede lograr resultados cercanos al Pareto óptimo de soluciones no dominadas, con una reducción del $75 \%$ en el tiempo de exploración en comparación con el proceso de búsqueda exhaustiva. Finalmente, se comparó el rendimiento de la metaheurística adoptada con el algoritmo genético de clasificación no dominado (NSGA-II) y el algoritmo evolutivo diferencial multiobjetivo (MODE).

Palabras clave: Redes de vehículos; 5G; mmWave; Computación evolutiva; Algoritmo de polinización de flores.

\section{Introduction}

The VANET (Vehicular Ad Hoc Networks) is a technology capable of providing the structure in which cars exchange information with each other and with different services, in order to increase the comfort and safety of the driver and pedestrians (Sheikh \& Liang, 2019). In this scenario, services such as collision avoidance systems, autonomous driving, realtime traffic routing, mobile entertainment, and many others, demand requirements, such as high data rates (Gbps) and ultra-low latency.

Interconnecting vehicular networks and any other latest technological devices represents a great challenge, especially due to intermittent links, dynamic architecture and the strict quality of service (QoS) requirements for VANETs (Ge et al., 2017).

However, the use of the mobile networks may be the key to accelerating the implementation of vehicular networks. The technology C-V2X (Cellular Vehicle-to-Everything) has attracted many interests from academic and industrial experts in information technology, automotive engineering and transportation engineering (Atallah et al., 2015; Chen at al., 2020), mainly because the mobile networks have a consolidated infrastructure, in addition to a wide coverage area. The $\mathrm{C}$ - $\mathrm{V} 2 \mathrm{X}$ is a 3rd Generation Partnership Project (3GPP) standard based on cellular systems. It is an alternative to 802.11p, adapted by IEEE to regulate access to the spectrum for dedicated short-range communications (DSRC), used in communication Vehicle-toVehicle (V2V) and Vehicle-to-Roadside (V2R).

In this context, the fifth generation of mobile networks based on millimeter waves (mmWave) is an excellent alternative for the implementation of C-V2X technology, since the goal of 5G is to reach Gigabytes per second and ultra-low latency (Attaran, 2021), in addition to $5 \mathrm{G}$ is currently available in many countries.

According to Tripathi et al. (2021) the Sub-6 GHz 5G standard only reach up $400 \mathrm{Mbit} / \mathrm{s}$, meanwhile, the 5G based on mmWave, best suited to vehicular communication requirements, is capable of providing higher peak data rate and ultra-low latency, this is possible because of the wide bandwidth available in is the band of spectrum, in addition, according to Mezzavilla et al. (2018), the mmWave technology allows the transmission of beams with much greater directionality than those previously used in cellular systems.

The millimeter waves is the band of spectrum with wavelengths between 10 millimeters (30 GHz) and 1 millimeter 
(300 GHz). Systems operating in these bands are attractive because of the large amounts of spectrum available in these higher frequency bands. On the other hand, spectrum below $6 \mathrm{GHz}$, which is considered for $3 \mathrm{GPP}$, has become very congested and individual bands are generally not available in contiguous blocks larger than $200 \mathrm{MHz}$ (Mezzavilla et al. 2018).

However, the mmWave signals are highly susceptible to blocking, this characteristic introduces serious challenges due to severe path and penetration losses (Zugno et al. 2020), moreover low propagation of signals at high frequency, which results in high variability of channel quality (Mezzavilla et al. 2018), and insufficient QoS level to guarantee the functionalities of services in VANETs, and thus compromise the safety of passengers, drivers, and pedestrians.

Crawley et al. (1998), defines QoS as a set of service requirements to be met by the network while transporting a flow, and can be measured in several ways: per flow, per link, or per node. Adjusts of parameters at different layers of the network stack, i.e., at the physical layer, MAC layer, and network layer, can improve the QoS (Adibi, 2010), besides, dynamic adjustment of these parameters, based certain scenarios, can keep the quality of service at acceptable levels and guarantee the perfect functioning of services in 5G mmWave. But, setting the optimal combination of these parameters can be costly, given the dimensionality of the problem, the size of the space of solutions, and the lack of a general model for the problem. However, the application of optimization algorithms can allow the achievement of the optimal solution for several instances of the same problem quickly.

Some scientific works have applied optimization algorithms to improve the quality of service in network communication. In Lacerda et al, (2018), the Non-dominated Sorting Genetic Algorithm II (NSGA-II) was used to explore the configuration space in the IEEE 1609.4 protocol, this approach showed a $90 \%$ reduction in the time needed to find the best parameters aiming at increasing the throughput, and reducing latency and packet loss. The same scenario simulated by Andrade et al. (2018), used the Multi-objective Discrete Differential Evolution (MODE) approach and achieved a 91\% reduction in time of exploration to find the best configuration.

Rawat et al. (2011) implemented an algorithm for dynamic adaptation of transmission power and contention window (CW) in ad-hoc vehicular networks. This scheme incorporates the Enhanced Distributed Channel Access (EDCA) mechanism of IEEE 802.11e and uses a joint approach to adapt transmission power at the physical (PHY) layer and QoS parameters at the medium access control (MAC) layer. The authors considered the urgency of messages, the instantaneous density of vehicles on the highway for the adaptation of transmission power, while only the collision rate was taken for the adaptation of the CW.

Studies by Lacerda et al. (2018) and Andrade et al. (2018), simulated mobility (SUMO, acronym for Simulation of Urban Mobility) and network (OMNeT++) components integrated via VEINS framework, to a realistic and flexible simulation.

Storck and Duarte-Figueiredo (2019), evaluated vehicular Internet-based video services traffic and Vehicle-to-Vehicle (V2V) communications. Simulations were performed through the Network Simulator NS-3, employing millimeter Wave (mmWave) communications. The metrics data transfer rate, transmission delay, and packet delivery ratio were analyzed. The results were evaluated in urban and rural scenarios. Although this approach evaluates and compares different scenarios, the authors did not apply any optimization algorithm to improve the evaluated metrics.

Sheng et al. (2018), proposed an intelligent 5G heterogeneous wireless network architecture which includes a reinforcement learning-based Dedicated Short Range Communication (DSRC) for Vehicle-to-Vehicle (V2V) and millimeter wave (mmWave) for Vehicle-to-Infrastructure (V2I) communications. Connectivity management components capable of optimizing the selection of the network based on the current situation were developed. This architecture developed connectivity management components able of optimizing the selection of the network based on the current situation. The results showed the effectiveness of this proposed architecture.

Rasheed and $\mathrm{Hu}$ (2021), propose a V2X network, which joins elements of Software Defined Network (SDN), 
Cognitive Radio (CR), and predictive routing techniques. The authors make use of 5G based on mmWave and of communications points that operates in the terahertz radio band ( $\mathrm{THz})$. The authors include deep learning algorithms, to predict the movement of nodes of VANET, and to find the best communication route. The proposed approach achieves ultra-high throughput and minimized delay, however, the approach uses algorithms that require powerful computational resources.

In that way, the problem of Quality of Service (QoS) in a $5 \mathrm{G}$ vehicle network based on mmWave is treated in this work. A framework to C-V2X simulation was developed using the network simulator NS-3, the urban mobility simulator SUMO, and a 5G mmWave module. The Multi-objective Flower Pollination Algorithm (MOFPA) was applied to explore the configuration of MAC layer parameters, to simultaneously optimize packet loss, latency, and throughput.

\section{Methodology}

The use of simulators is an excellent alternative for the study of communication networks and their behavior under different scenarios. Some of them, open-source, are highly regarded in the scientific community due to the fidelity of the results. Thus, to simulate a $5 \mathrm{G}$ mmWave vehicular network, this work developed a framework based on the open-source network simulator NS-3, on the mobility simulator, SUMO, and on the mmWave module developed by Mezzavilla et al (2018).

The module mmWave includes statistical channel models and incorporates real measurements based on the NS-3 Long Term Evolution (LTE), the physical (PHY) and medium access control (MAC) layers are customizable and integrable with algorithms. The architecture of the framework for vehicular network optimization can be seen in Figure 1.

Figure 1. Framework architecture for $5 \mathrm{G}$ mmWave vehicular network optimization.

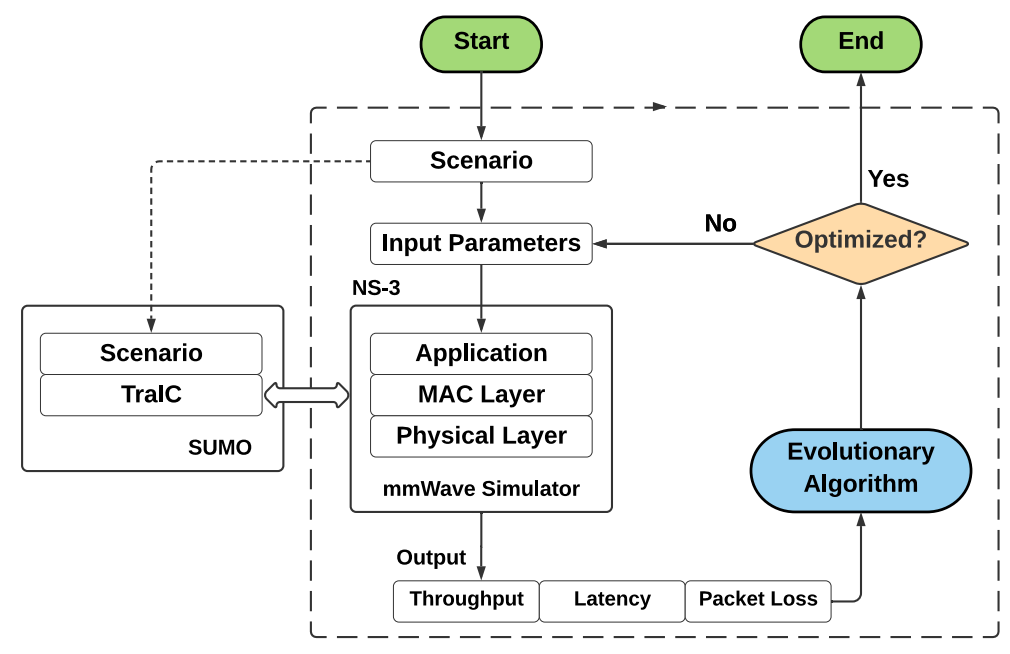

Source: Authors.

Thus the framework implemented is able to simulate road traffic and the interaction between vehicles through $\mathrm{C}$-V2X technology in urban and rural scenarios with different traffic densities.

To reach the objective of this work, four parameters of the EDCA were explored, once the IEEE 802.11p standard introduces the EDCA mechanism in the MAC layer to support the quality of service in VANETs (Chang et al., 2016). To complement the MAC layer adjustment parameters, the transmission power of the DSRC also was explored. The four parameters used and the space of possible values configuration are depicted in Table 1. 
Table 1. Exploration space for mmWave vehicular network.

\begin{tabular}{ll}
\hline Parameter & Discrete Value \\
\hline Subframe Period & $\mathrm{P} 1=\{75,100,150,200\}$ \\
Symbols per Frame & $\mathrm{P} 2=\{16,24,32,40\}$ \\
HARQ process & $\mathrm{P} 3=\{1,2,4\}$ \\
TX power $(\mathrm{dBm})$ & $\mathrm{P} 4=\{5,10,15,20,25,30,35,40,45,50\}$ \\
\hline
\end{tabular}

Source: Authors.

Generally, network simulators model all the protocol stack as well as applications running over the network, and because of the complexity of simulating, a single simulation of a mmWave based vehicular network can take a long time, requiring the application of strategies to minimize time cost. The simulation of the proposed approach considered an urban scenario with moderate traffic.

Optimization problems, such as the one discussed in this paper, are often solved by using a metaheuristic that mimics animals' behaviors or physics phenomena. Thus, among several existing metaheuristics, we chose one with proven efficiency in solving engineering problems. Kayabekir et al. (2017) list several successful applications of the Flower Pollination Algorithm, implemented by other authors in chemical engineering, civil engineering, energy and power systems, mechanical engineering, electronical, and communication engineering and computer science. The authors mention that, in addition to being applied to optimization problems, the algorithm can also be applied in data mining such as feature selection and classifications.

In this way, the Multi-objective Flower Pollination Algorithm, proposed by Yang et al. (2013), was applied to search for optimal combinations for the problem addressed in this work. Algorithm 1 illustrates the pseudocode for MOFPA.

Algorithm 1. The MOFPA Pseudocode.

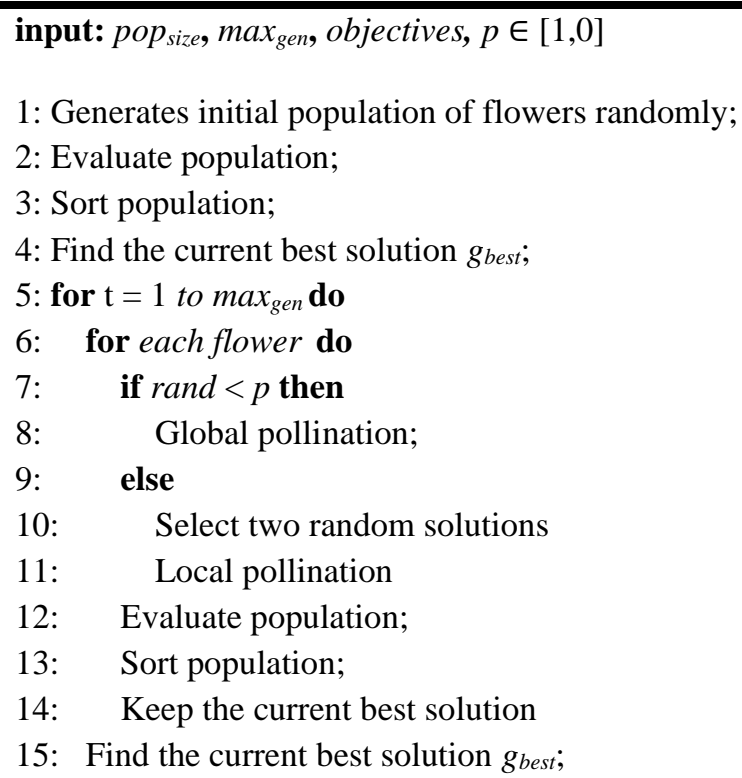

Source: Adapted from Yang et al. (2013).

The MOFPA, is based on swarm intelligence and mimics the dynamics of flowers pollination, where the male gamete (pollen) is transferred to stigma, to fertilize the female gamete. The pollen travels from one flower to another in two ways: Biotic and Abiotic. The first occurs when an animal carries pollen, and the second occurs when the pollen is transported through air, water, or gravity.

The input parameters of the algorithm are: Population size pop $_{\text {size }}$, maximum number of generations $\max _{\text {gen }}$, the 
variable objectives, which represents the objective functions of the problem, and the probability $p$, which controls the alternation between local and global search. After randomly initializing the individuals of population, line 1, the MOPFA, calculates the objective functions of each individual, line 2 , and then, in line 3 , sorts the individuals, using non-dominance criteria. Then, the algorithm determines the best solution applying distance calculation metrics, line 4 . The conditional structure, line 7, randomly determines if the pollination process will be global or local. In the case of biotic pollination (global search) the algorithm takes into account the best individual $g_{\text {best }}$ of the current generation and calculates equation 1 .

$$
x_{i}^{t+1}=x_{i}^{t}+\gamma\left(x_{i}^{t}+g_{\text {best }}\right)
$$

To generate the pseudo-random movement of pollen, based on the Lévy flight, MOFPA uses the equations 2,3, and 4 .

$$
\begin{gathered}
L=\frac{\lambda \Gamma(\lambda) \sin \left(\frac{\pi \lambda}{2}\right)}{\pi},\left(s>>s_{0}>s\right) \\
S=\frac{U}{|V|^{\frac{1}{\lambda}}}, U \sim N\left(0, \sigma^{2}\right), V \sim N(0,1)
\end{gathered}
$$

The variables $\mathrm{U}$ and $\mathrm{V}$, from equation 3 , are samples from a normal distribution.

$$
\sigma=\left[\frac{\gamma(1+\lambda)}{\lambda \Gamma((1+\lambda) / 2)} \cdot \frac{\sin \left(\frac{\pi \lambda 2}{2}\right)}{2^{(\lambda-1) / 2}}\right]^{1 / \lambda}
$$

In abiotic pollination (local search) the algorithm randomly selects two individuals, $x_{j}$ and $x_{k}$, randomly generates the value of $\varepsilon \in[1,0]$, and finally calculates the equation 5. To modify the behavior of MOFPA, the values of the Lévy distribution index $\lambda$ and scale factor $\gamma$ can be adjusted.

$$
x_{i}^{t+1}=x_{i}^{t}+\varepsilon\left(x_{i}^{t}+x_{k}^{t}\right)
$$

\section{Results and Discussion}

With the purpose of to find out the ideal population size and the optimal number of generations to solve the proposed approach, the relationship between population size and the number of simulations was analyzed. In all simulations five population sizes $(10,20,30,40$, and 50 individuals) were used.

The exhaustive search requires 480 simulations to solve this problem, but the Figure 2, depicts that proposed approach requires a smaller number of simulations.

To explore the ideal population size, multi-objective metrics must be calculated. The Hypervolume (HV), Generational Distance (GD), Inverted Generational Distance (IGD), and Spacing (SP), consider three aspects: convergence, scattering, and distance from the true Pareto Front (PF) of the problem.

The HV measures the size of the portion of the objective space that is dominated by solutions as a group (Santos and Xavier, 2018). Thus, high values of HV indicate that the set of solutions dominates a large part of the objective space. The GD and IGD measure how far the Pareto Front generated by the algorithm is from the true Pareto Front of the problem. When PF is unknown, a reference set is used to estimate proximity. Low values for GD/IGD represent the better performance of results (Santos \& Xavier, 2018). The metric SP denotes how evenly distributed PF solutions are in the objectives exploration space. Values close to 0 indicate a more distributed and sparse set of non-dominated solutions. 
Figure 2. Number of simulations as a function of the numbers of generations for different population size.

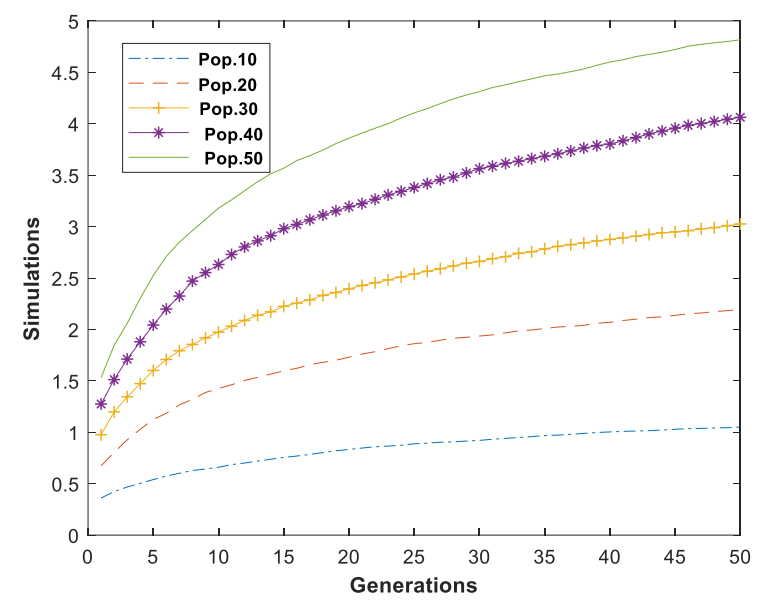

Source: Authors.

The source code of these indicators can be found in PlatEMO, a platform for evolutionary multi-objective optimization (Tian et al., 2017). The average value of the metrics and the standard deviation are shown in Table 2.

Table 2. Average of metrics and its standard deviation over 50 iterations.

\begin{tabular}{|c|c|c|c|c|}
\hline & \multicolumn{4}{|c|}{ Multi-objective metric ( $\sigma$ ) } \\
\hline & HV $\left(\mathbf{1 0}^{-1}\right)$ & GD & IGD & SP \\
\hline 10 & $1.28(0.45)$ & $2.83(4.79)$ & $22.59(2.69)$ & $9.87(7.79)$ \\
\hline 20 & $1.55(0.33)$ & $1.49(2.60)$ & $13.52(1.47)$ & $6.44(4.84)$ \\
\hline 30 & $1.81(0.31)$ & $1.21(1.88)$ & $9.82(1.44)$ & $3.22(6.04)$ \\
\hline 40 & $2.05(0.34)$ & $1.05(2.17)$ & $8.97(1.15)$ & $2.33(1.72)$ \\
\hline 50 & $2.33(0.25)$ & $0.92(2.23)$ & $7.78(1.59)$ & $1.84(3.87)$ \\
\hline
\end{tabular}

Source: Authors.

From Table 2, it is possible to verify that all metrics tend to improve as the population size increases. Thus, the behavior of the value of these metrics, for the purpose of defining the ideal population size, is inconclusive. Therefore, now we are going to analyze the minimum objective values as a function of generation. The graphs in Figures 3, 4, and 5, represents statistical results of MOFPA performance in optimization of packet loss, latency and throughput, respectively. The graphs were generated from averages of 30 simulations to different population sizes over 50 generations.

The graphs in Figures 3 and 4 represent statistical results of packet loss and latency. In both cases, population sizes with more individuals achieved better results to the problem addressed. 
Figure 3. Evolution in terms of packet loss as a function of the numbers of generations for different population size.

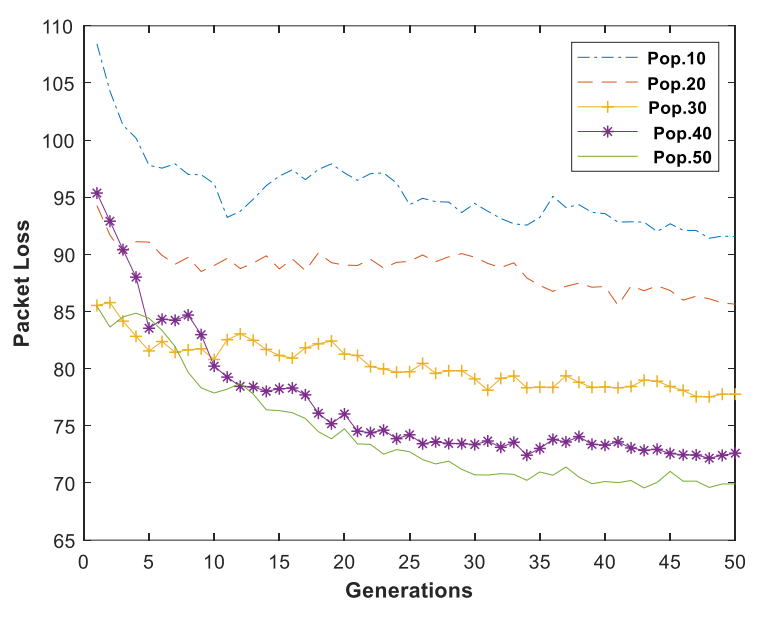

Source: Authors.
Figure 4. Evolution in terms of latency as a function of the numbers of generations for different population size.

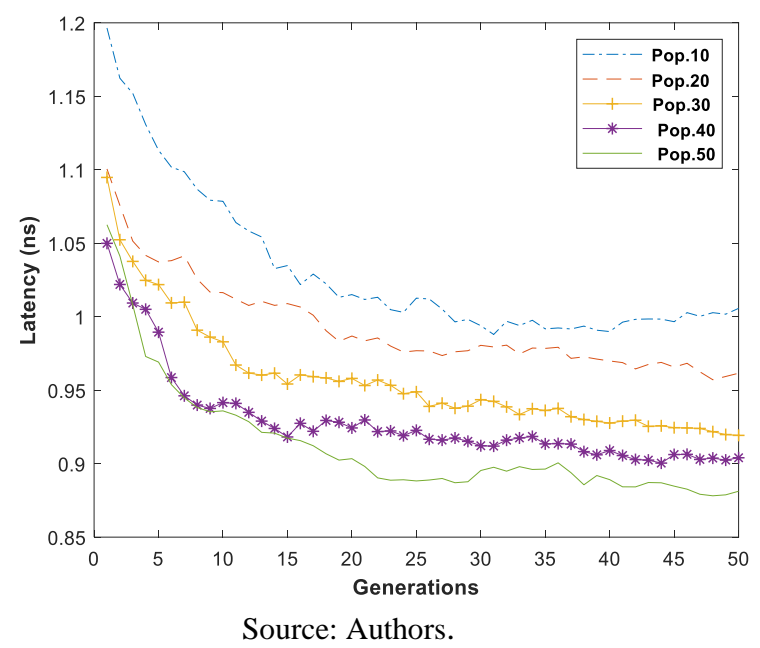

Although the proposed approach aims to optimize packet loss, latency, and throughput simultaneously, we can see in Figure 5 that the MOFPA failed to maximize throughput.

The Figure 5 also shows that the population sizes of 30 and 40 individuals reached very similar throughput values, while a population size of 50 individuals had the worst result. This is explained by the spreading of solutions in the search space. The exhaustive search (see Figure 6) shows the solutions divided in three zones well-delimited.

Figure 5. Evolution in terms of throughput as a function of the numbers of generations for different population size.

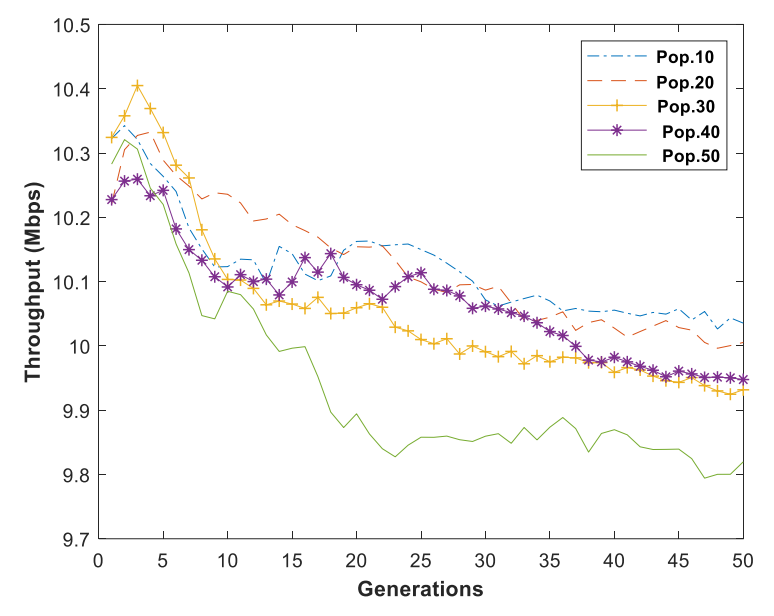

Source: Authors.
Figure 6. Exploratory analysis of the solutions space and pareto of proposed approach.

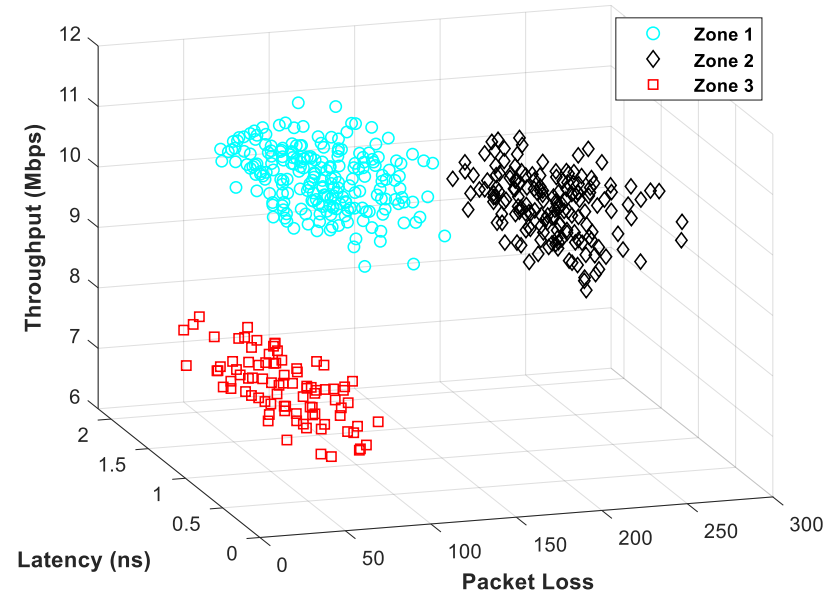

Source: Authors. 
We can see that Zone 1 has the best solutions for throughput, Zone 2 has good solutions to minimize latency and Zone 3 has the best solutions for packet loss reduction. Thus, the pike values of throughput, in Zone 1 (represented by the blue circles), are in a different region from where the best solutions for packet loss and latency are located. Therefore, due to the characteristics of the problem, it is not possible to optimize the three parameters simultaneously, in other words, maximizing throughput implies increasing packet loss, latency, or both. In a C-V2X network several services with different characteristics can be available, such as platoon control, video streaming, alert and security messages, etc. So the best Zone depends on the requirements of service and what metrics must be prioritized.

In the graph in Figure 6, we can see that the exhaustive search found regions that can maintain satisfactory QoS levels without optimizing the three parameters simultaneously. Furthermore, we can see in Figures 3, 4, and 5, which population size greater than 30 and less than 40 leads MOFPA to find solutions within these regions. Thus, we can say that a population size with 35 individuals is enough to solve the problem addressed in this paper.

The simulations with a population size of 35 individuals showed that the PF converges rapidly up to around 30 iterations. After that, the algorithm finds a local minimum, and remains there until the stopping criterion, without signific ant changes in solutions found. Given the above, we can conclude that for the problem addressed, MOFPA generates near-optimal solutions, with a population size of 35 individuals and a stopping criterion for 25 generations.

The exhaustive search requires 480 iterations to find the optimal solution to the problem addressed, on the other hand, the approach proposed for $p p_{\text {size }}=35$ and max $_{\text {gen }}=25$, the number of simulations needed is 120 , which means, $75 \%$ reduction in time required. Table 3 shows the number of simulations and time needed for each technique.

Table 3. Comparison of the MOFPA with the exhaustive search.

\begin{tabular}{lcc}
\hline Mechanism & Number of Simulations & Total Time (min) \\
\hline Exhaustive Search & 480 & 24000 \\
MOFPA $\left(\right.$ pop $_{\text {size }}=\mathbf{3 5}{\left.\text {, } \boldsymbol{m a x}_{\text {gen }} \mathbf{= 2 5}\right)}^{25}$ & 120 & 6000 \\
\hline
\end{tabular}

Source: Authors.

In order to evaluate the performance of MOFPA, the problem formulated was applied to the NSGA-II (Deb et al. 2002) and the MODE (Zang, 2009). Both techniques have a long history of application in optimization problems. For comparison purposes, the simulation parameters were, $\operatorname{pop}_{\text {size }}=35$ and $\max _{g e n}=25$. Table 4 summarizes the results obtained considering the number of simulations and time needed to find the best solutions.

Table 4. Comparison of the MOFPA with other metaheuristics.

\begin{tabular}{lccc}
\hline Mechanism & Number of Simulations & Total Time (min) & Rank \\
\hline Exhaustive Search & 480 & 24000 & \\
MOFPA $\left(\right.$ pop $_{\text {size }}=\mathbf{3 5}$, max $\left._{\text {gen }}=\mathbf{2 5}\right)$ & 120 & 6000 & 1 \\
NSGA-II $\left(\right.$ pop $_{\text {size }}=\mathbf{3 5}$, max $\left._{\text {gen }}=\mathbf{2 5}\right)$ & 160 & 8000 & 2 \\
MODE $\left(\right.$ pop $_{\text {size }}=\mathbf{3 5}$ max $\left._{\text {gen }}=\mathbf{2 5}\right)$ & 190 & 9500 & 3 \\
\hline
\end{tabular}

Source: Authors.

\section{Conclusion}

In this work, a VANET C-V2X was simulated in an urban environment, with the objective of optimizing throughput, latency and packet loss simultaneously, through adjustments in MAC layer parameters, and DSRC transmission power. The 5G mmWave vehicular network was simulated with a framework built with NS-3, SUMO, and a 5G mmWave module. 
The exhaustive search showed that the solutions to the problem addressed were grouped in well-defined Zones, in this way, maximizing throughput and simultaneously minimizing latency and packet loss would not be possible. However, some regions of the search space present near-optimal solutions, ensuring a satisfactory level of QoS.

The MOFPA metaheuristics was used to find the optimal combinations for the MAC layer parameters and DSRC transmission power. The Population size of 35 individuals, and stopping criterion for 25 generations, was enough for the metaheuristic achieve near-optimal solutions. Simulations for these configurations show solutions with throughput greater than $10 \mathrm{Mbps}$, latency less than 1ns, packet loss at acceptable levels, and reduced the time cost by $75 \%$ compared to exhaustive search.

For performance comparison purposes, NSGA-II and MODE were applied to the same problem for pop $_{\text {size }}=35$ and $\max _{\text {gen }}=25$. The results showed that both algorithms require less time cost than exhaustive searching to find optimal solutions. However, the performance of the Multi-objective Flower Pollination Algorithm was superior to the three techniques analyzed.

For future work, it is intended to optimize the adjustable parameters of MOFPA in order to improve its performance in optimization problems applied to vehicular networks with various scenarios.

\section{References}

Adibi, S., Jain, R., Parekh, S., \& Tofighbakhsh, M. (Eds.). (2010). Quality of service architectures for wireless networks: Performance metrics and management. Hershey. Information Science Reference.

Andrade, H. G. V., Rios, M. F. R., Lima, R. N., Lacerda, H. F., \& Silva-Filho, A. G. (2018). Multi-objective approaches to improve QoS in vehicular ad-hoc networks. Proceedings of the 8th ACM Symposium on Design and Analysis of Intelligent Vehicular Networks and Applications - DIVANet'18.

Atallah, R. F., Khabbaz, M. J., \& Assi, C. M. (2015). Vehicular networking: A survey on spectrum access technologies and persisting challenges. Vehicular Communications, 2(3), 125-149. https://doi.org/10.1016/j.vehcom.2015.03.005

Attaran, M. (2021). The impact of $5 \mathrm{G}$ on the evolution of intelligent automation and industry digitization. Journal of Ambient Intelligence and Humanized Computing, 1-17. https://doi.org/10.1007/s12652-020-02521-x

Chang, C.-Y., Yen, H.-C., \& Deng, D.-J. (2016). V2V QoS Guaranteed Channel Access in IEEE 802.11p VANETs. IEEE Transactions on Dependable and Secure Computing, 13(1), 5-17. https://doi.org/10.1109/tdsc.2015.2399912

Chen, S., Hu, J., Shi, Y., Zhao, L., \& Li, W. (2020). A vision of C-V2X: Technologies, field testing, and challenges with Chinese development. IEEE Internet of Things Journal, 7(5), 3872-3881. https://doi.org/10.1109/jiot.2020.2974823

Crawley, E., Nair, R., Rajagopalan, B., \& Sandick, H. (1998). A framework for QoS-based routing in the internet. RFC Editor.

Deb, K., Pratap, A., Agarwal, S., \& Meyarivan, T. (2002). A fast and elitist multiobjective genetic algorithm: NSGA-II. IEEE Transactions on Evolutionary Computation : A Publication of the IEEE Neural Networks Council, 6(2), 182-197. https://doi.org/10.1109/4235.996017

Ge, X., Li, Z., \& Li, S. (2017). 5G Software Defined Vehicular Networks. IEEE Communications Magazine, 55(7), 87-93. https://doi.org/10.1109/mcom.2017.1601144

Kayabekir, A. E., Bekdaş, G., Nigdeli, S. M., \& Yang, X.-S. (2018). A comprehensive review of the flower pollination algorithm for solving engineering problems. In Nature-Inspired Algorithms and Applied Optimization (pp. 171-188). Springer International Publishing.

Lacerda, H. F., Andrade, H. G. V., \& Silva-Filho, A. G. (2018). Improving QoS in Vehicular ad-hoc Networks using a multi-objective optimization algorithm. 2018 IEEE Symposium on Computers and Communications (ISCC).

Mezzavilla, M., Zhang, M., Polese, M., Ford, R., Dutta, S., Rangan, S., \& Zorzi, M. (2018). End-to-end simulation of 5G mmWave networks. IEEE Communications Surveys \& Tutorials, 20(3), 2237-2263. https://doi.org/10.1109/comst.2018.2828880

Rasheed, I., \& Hu, F. (2021). Intelligent super-fast Vehicle-to-Everything 5G communications with predictive switching between mmWave and THz links. Vehicular Communications, 27(100303), 100303. https://doi.org/10.1016/j.vehcom.2020.100303

Rawat, D. B., Popescu, D. C., Yan, G., \& Olariu, S. (2011). Enhancing VANET performance by joint adaptation of transmission power and contention window size. IEEE Transactions on Parallel and Distributed Systems: A Publication of the IEEE Computer Society, 22(9), 1528-1535. https://doi.org/10.1109/tpds.2011.41

Santos, T., \& Xavier, S. (2018). A Convergence Indicator for Multi-Objective Optimisation Algorithms. TEMA. Tendencias Em Matematica Aplicada e Computacional, 19(3), 437. https://doi.org/10.5540/tema.2018.019.03.437

Sheikh, M. S., \& Liang, J. (2019). A comprehensive survey on VANET security services in traffic management system. Wireless Communications and Mobile Computing, 2019, 1-23. https://doi.org/10.1155/2019/2423915 
Research, Society and Development, v. 11, n. 1, e33911125020, 2022

(CC BY 4.0) | ISSN 2525-3409 | DOI: http://dx.doi.org/10.33448/rsd-v11i1.25020

Sheng, Z., Pressas, A., Ocheri, V., Ali, F., Rudd, R., \& Nekovee, M. (2018). Intelligent 5G vehicular networks: An integration of DSRC and mmWave communications. 2018 International Conference on Information and Communication Technology Convergence (ICTC).

Storck, C., \& Duarte-Figueiredo, F. (2019). A 5G V2X ecosystem providing Internet of vehicles. Sensors (Basel, Switzerland), 19(3), 550. https://doi.org/10.3390/s19030550

Tian, Y., Cheng, R., Zhang, X., \& Jin, Y. (2017). PlatEMO: A MATLAB platform for evolutionary multi-objective optimization. IEEE Computational Intelligence Magazine, 12(4), 73-87. https://doi.org/10.1109/mci.2017.2742868

Tripathi, S., Sabu, N. V., Gupta, A. K., \& Dhillon, H. S. (2021). Millimeter-wave and terahertz spectrum for 6G wireless. In Computer Communications and Networks (pp. 83-121). Springer International Publishing.

Yang, X.-S., Karamanoglu, M., \& He, X. (2013). Multi-objective flower algorithm for optimization. Procedia Computer Science, 18, 861-868. https://doi.org/10.1016/j.procs.2013.05.251

Zhang, M., Zhao, S., \& Wang, X. (2009). Multi-objective evolutionary algorithm based on adaptive discrete Differential Evolution. 2009 IEEE Congress on Evolutionary Computation.

Zugno, T., Drago, M., Giordani, M., Polese, M., \& Zorzi, M. (2020). Toward standardization of millimeter-wave vehicle-to-vehicle networks: Open challenges and performance evaluation. IEEE Communications Magazine, 58(9), 79-85. https://doi.org/10.1109/mcom.001.2000041 